\title{
DLG5 connects cell polarity and Hippo signaling protein networks by linking PAR-1 with MST1/2
}

\author{
Julian Kwan, ${ }^{1,2,7}$ Anna Sczaniecka, ${ }^{3,7}$ Emad Heidary Arash, ${ }^{1,4}$ Liem Nguyen, ${ }^{3}$ Chia-Chun Chen, ${ }^{3}$ \\ Srdjana Ratkovic, ${ }^{2,5}$ Olga Klezovitch, ${ }^{3}$ Liliana Attisano, ${ }^{1,4}$ Helen McNeill, ${ }^{2,5}$ Andrew Emili, ${ }^{1,2}$ \\ and Valeri Vasioukhin ${ }^{3,6}$ \\ ${ }^{1}$ Donnelly Centre for Cellular and Biomolecular Research, ${ }^{2}$ Department of Molecular Genetics, University of Toronto, Toronto, \\ Ontario M5S 3E1, Canada; ${ }^{3}$ Division of Human Biology, Fred Hutchinson Cancer Research Center, Seattle, Washington 98109, \\ USA; ${ }^{4}$ Department of Biochemistry, University of Toronto, Toronto, Ontario M5S 1A8, Canada; ${ }^{5}$ Lunenfeld-Tanenbaum Research \\ Institute, Mt. Sinai Hospital, Toronto, Ontario M5G 1X5, Canada; ${ }^{6}$ Department of Pathology, Institute for Stem Cell and \\ Regenerative Medicine, University of Washington, Seattle, Washington 98195, USA
}

\begin{abstract}
Disruption of apical-basal polarity is implicated in developmental disorders and cancer; however, the mechanisms connecting cell polarity proteins with intracellular signaling pathways are largely unknown. We determined previously that membrane-associated guanylate kinase (MAGUK) protein discs large homolog 5 (DLG5) functions in cell polarity and regulates cellular proliferation and differentiation via undefined mechanisms. We report here that DLG5 functions as an evolutionarily conserved scaffold and negative regulator of Hippo signaling, which controls organ size through the modulation of cell proliferation and differentiation. Affinity purification/mass spectrometry revealed a critical role of DLG5 in the formation of protein assemblies containing core Hippo kinases mammalian ste20 homologs 1/2 (MST1/2) and Par-1 polarity proteins microtubule affinity-regulating kinases 1/2/3 (MARK1/2/3). Consistent with this finding, Hippo signaling is markedly hyperactive in mammalian $\mathrm{Dlg} 5^{-/-}$tissues and cells in vivo and ex vivo and in Drosophila upon $d \lg 5$ knockdown. Conditional deletion of Mst1/2 fully rescued the phenotypes of brain-specific Dlg 5 knockout mice. Dlg5 also interacts genetically with Hippo effectors Yap1/Taz. Mechanistically, we show that DLG5 inhibits the association between MST1/2 and large tumor suppressor homologs 1/2 (LATS1/2), uses its scaffolding function to link MST1/2 with MARK3, and inhibits MST1/2 kinase activity. These data reveal a direct connection between cell polarity proteins and Hippo, which is essential for proper development of multicellular organisms.
\end{abstract}

[Keywords: protein complex; interaction network; functional proteomics; cell polarity; Hippo signaling; Drosophila development; mammalian development]

Supplemental material is available for this article.

Received May 19, 2016; revised version accepted December 7, 2016.

Cell polarity mechanisms are pivotal for the proper integration of individual cells into organs and tissues (Bryant and Mostov 2008). Disruption of cell polarity results in prominent developmental defects, abnormal differentiation, and, in some cases, uncontrolled proliferation and cancer (Martin-Belmonte and Perez-Moreno 2012). The exact molecular mechanisms connecting cell polarity with cell proliferation and differentiation remain largely unknown.

Discs large homolog 5 (Dlg5) encodes a large membrane-associated guanylate kinase (MAGUK) multidomain protein suggestive of a scaffolding and/or signaling function containing CARD, a coiled-coil domain, four

\footnotetext{
${ }^{7}$ These authors contributed equally to this work. Corresponding authors: vvasiouk@fredhutch.org, andrew.emili@utoronto.ca

Article is online at http://www.genesdev.org/cgi/doi/10.1101/gad.284539. 116.
}

PDZ domains, and SH3 and GUK domains (Nechiporuk et al. 2007). Genetic variations in human DLG5 are associated with inflammatory bowel and Crohn's disease (Stoll et al. 2004). Loss of DLG5 expression has also been noted in prostate and bladder cancers, where it results in activation of cell invasion and metastasis (Tomiyama et al. 2015; Zhou et al. 2015). DLG5 has been shown to regulate Hedgehog signaling (Chong et al. 2015), while, in Drosophila, dlg5 is an essential gene necessary for biogenesis of primordial germ cells (Reilly et al. 2015).

We showed previously that $D \lg 5^{-/-}$mice display prominent defects in apical-basal cell polarity and develop

\footnotetext{
(C) 2016 Kwan et al. This article is distributed exclusively by Cold Spring Harbor Laboratory Press for the first six months after the full-issue publication date (see http://genesdev.cshlp.org/site/misc/terms.xhtml). After six months, it is available under a Creative Commons License (Attribution-NonCommercial 4.0 International), as described at http://creativecommons.org/licenses/by-nc/4.0/.
} 
brain hydrocephalus, synaptogenesis defects, emphysema-like lungs, and kidney cysts (Nechiporuk et al. 2007, 2013; Wang et al. 2014). Dlg5 is necessary for the proper maintenance and proliferation of progenitor cells in vivo, and $D \lg 5^{-/-}$pups and adult mice are correspondingly much smaller than their wild-type littermates. The mechanisms responsible for Dlg5-mediated regulation of intracellular signaling pathways governing cell proliferation and differentiation remained undiscovered.

The Hippo tumor suppressor pathway plays a pivotal role in regulating many biological processes, including cell proliferation, survival, differentiation, and organ size control (Mo et al. 2014). The conserved protein kinases MST1 (mammalian ste20 homolog 1) and MST2 are orthologs of the Drosophila hippo (hpo) kinase. MST1/2 play a key role in activation of the downstream kinases LATS $1 / 2$ (large tumor suppressor homologs 1/2), which in turn phosphorylate and inactivate the transcriptional coactivator proteins YAP and TAZ, inhibiting their nuclear localization and promoting their degradation (Barry and Camargo 2013; Yu and Guan 2013). Thus, the primary function of Hippo signaling is suppression of the transcriptional activity of YAP/TAZ. Upstream mechanisms responsible for the regulation of MST1/2 kinases are critical but poorly understood aspects of the Hippo signaling system.

MST1/2 have several documented binding partners, which include the SARAH domain (Salvador, RASSF, Hippo)-containing proteins SAV1 and RASSF proteins 1-6. SAV1 is a scaffold protein, which promotes MST1/2-mediated phosphorylation and activation of LATS1/2, while the different RASSF homologs appear to play both activating and inhibitory roles (Ni et al. 2013). MST1/2 and the entire Hippo pathway are highly sensitive to the changes in apical-basal cell polarity; however, the mechanisms physically connecting Hippo kinases to cell polarity are not well understood.

To explore DLG5-mediated regulation of mammalian progenitor cell maintenance and morphogenesis, we performed an extensive and unbiased affinity purification/ mass spectrometry (AP/MS) screen to identify proteins physically associated with DLG5 in human cell culture. We found that DLG5 interacts directly with MST1/2 kinases and thereby negatively regulates the activity of the Hippo signaling pathway. DLG5 serves as a scaffolding protein that recruits microtubule affinity-regulating kinase 3 (MARK3) to MST1/2, promoting hyperphosphorylation of MST1/2. Overall, we characterize a novel and direct mechanistic connection between cell polarity proteins and Hippo signaling and demonstrate the significance of this connection during metazoan embryonic development.

\section{Results}

Systematic proteomic analyses reveal physical connectivity between DLG5, apical-basal cell polarity, and Hippo pathway protein networks

To gain insights into the molecular mechanisms underlying DLG5-mediated regulation of tissue morphogene- sis, we initiated our AP/MS study with $\mathrm{N}$-terminal epitope-tagged DLG5 expressed in human HEK293T cells (Fig. 1A). Precision MS analysis established prominent, specific, and reproducible copurification of DLG5 with MST1/2 and Salvador (SAV1), core components of the Hippo signaling pathway (Fig. 1A,B). While our AP/ MS experiments identified multiple previously unreported DLG5-interacting proteins, notably MARK1/2/3 (Fig. 1A; Supplemental Table S1), we were most intrigued by the interaction between DLG5 and MST1/2 kinases, which play a central role in the canonical Hippo signaling pathway.

To examine how DLG5 interacts with MST1/2 and associated cofactors, we performed comparative AP/MS analyses of epitope-tagged wild-type and kinase-dead variants of MST1 and MST2 as well as 19 other Hippo signaling and cell polarity-related factors, including all other human SARAH domain-containing proteins (Fig. 1A; Supplemental Table S2). Strikingly, tagged but otherwise wild-type MST1/2 showed relatively weak binding (i.e., low copurifying spectral counts), whereas kinase-dead MST1/2 showed a much stronger association with DLG5 (Fig. 1B). Conversely, the previously reported interaction between MST1/2 and the striatin-interacting phosphatase and kinase (STRIPAK) complex (Couzens et al. 2013; Hauri et al. 2013) was readily recovered with the wild-type MST1/2 (Fig. 1B) but was abrogated for kinasedead MST1/2. None of the other cell polarity proteins examined showed an interaction with MST1/2, although we did find that RASSF6 interacted stably with apical-basal cell polarity proteins. Moreover, neither MST1/2 nor SAV1 copurified with tagged DLG1 or DLG2, which are distantly related to DLG5 (Nechiporuk et al. 2007), indicating that the DLG5-MST1/2 interaction is specific for DLG5 and not a feature of all members of the DLG protein family. Conversely, each SARAH domain-containing RASSF protein strongly interacted with MST1/2, but we did not detect an association of a RASSF protein with any other SARAH proteins, demonstrating that MST1/2 participates in different, possibly mutually exclusive, multiprotein complexes, as proposed by the Gstaiger laboratory (Hauri et al. 2013). Our data also imply another distinct MST1/2 regulatory protein complex containing DLG5.

Specific protein binding between DLG5 and MST1/2 was first confirmed by coexpression and coimmunoprecipitation (co-IP) experiments (Supplemental Fig. S1). Next, we assessed interactions between endogenous proteins under physiological conditions. For these experiments, we decided to use primary embryonic neural progenitor cells (NPCs) because Dlg5 $5^{-/-}$embryos display prominent polarity and proliferation defects in these cells, causing hydrocephalus in Dlg5 $5^{-/}$mice (Nechiporuk et al. 2007; Chang et al. 2010). We performed co-IP experiments using antibodies raised against either DLG5 or MST1/2 as well as lysate from primary murine NPCs isolated from embryonic day 12.5 (E12.5) brains of both wild-type and Dlg5 ${ }^{-/-}$ embryos. As expected, co-IP with anti-Dlg5, but not negative control IgG antibodies, pulled down MST1/2 from wild-type, but not $D \lg 5^{-/-}$, cell lysates (Fig. 1C). 
A

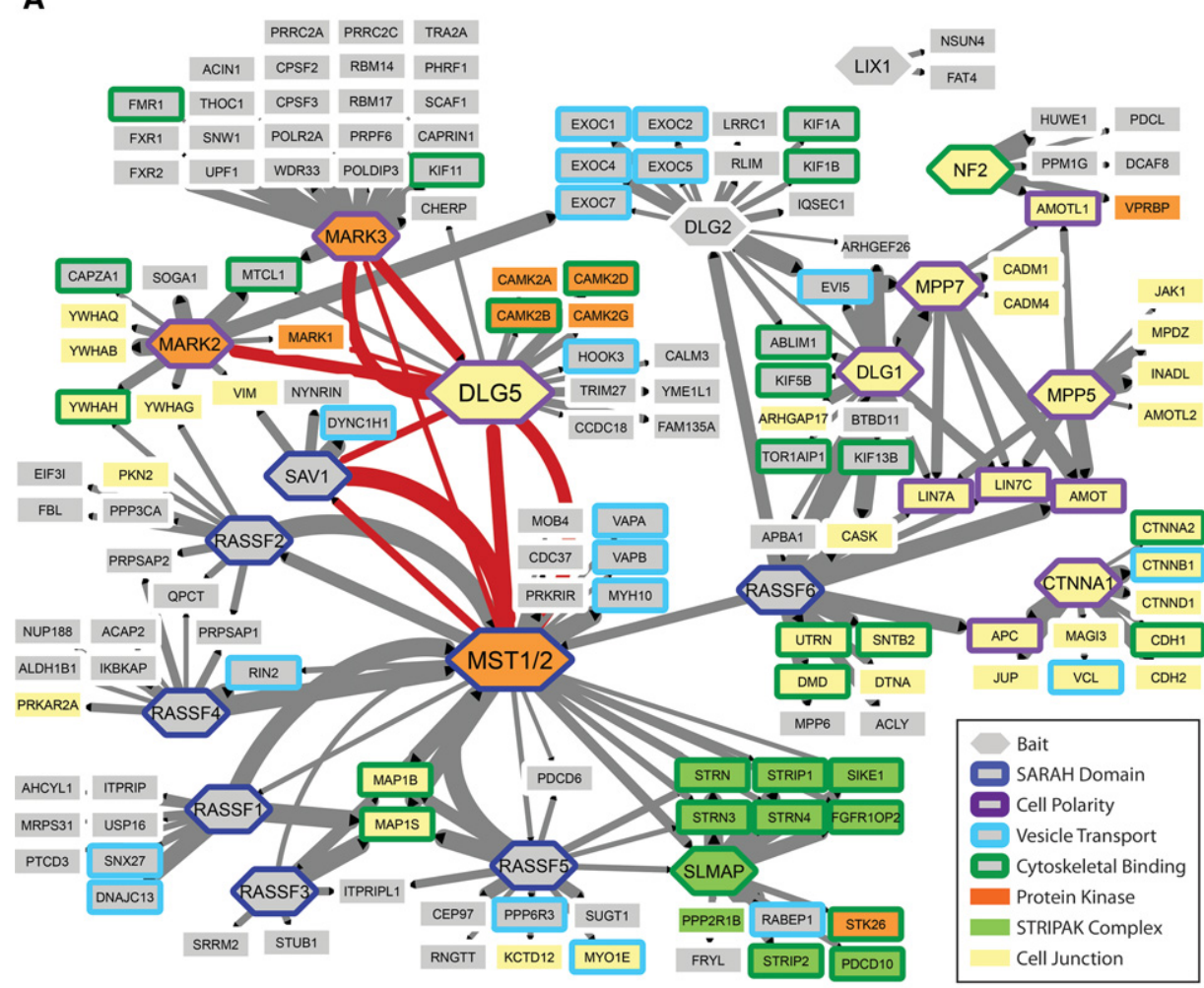

B

C

\begin{tabular}{|c|c|c|c|c|c|c|c|}
\hline \multirow[b]{2}{*}{ Protein ID } & \multicolumn{6}{|c|}{ Baits } & \\
\hline & DLG5 & MST1-KD & MST2-KD & MST1 & MST2 & RASSF5 & \\
\hline DLG5 & 326 & 7 & 39 & 0 & 1 & 0 & \\
\hline MARK3 & 28 & 0 & 5 & 0 & 0 & 0 & \\
\hline SAV1 & 16 & 14 & 27 & 14 & 32 & 0 & \\
\hline MST1 & 5 & 411 & 59 & 284 & 79 & 99 & $\frac{\text { क }}{8}$ \\
\hline MST2 & 27 & 290 & 638 & 220 & 485 & 208 & ○్ \\
\hline RASSF5 & 0 & 3 & 6 & 4 & 11 & 217 & ప \\
\hline SLMAP & 0 & 0 & 0 & 16 & 20 & 5 & 8 \\
\hline STRN & 0 & 0 & 0 & 14 & 11 & 10 & క \\
\hline STRN3 & 0 & 0 & 0 & 14 & 9 & 2 & \\
\hline \begin{tabular}{|l|} 
STRN4 \\
\end{tabular} & 0 & 0 & 0 & 3 & 8 & 2 & \\
\hline SIKE1 & 0 & 0 & 0 & 5 & 6 & 3 & \\
\hline
\end{tabular}

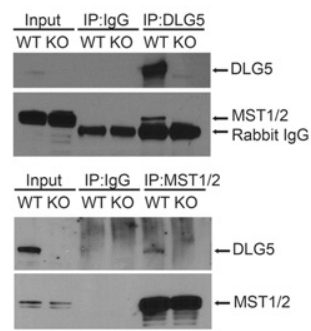

Figure 1. Unbiased AP/MS centered on DLG5 reveals a connection between DLG5 and the Hippo signaling pathway. $(A)$ Network representation of high confidence physical interactions. Highlighted are AP/MS bait proteins (hexagons), stably interacting proteins (rectangles) annotated as SARAH domain-containing proteins of the Hippo pathway (blue), cell polarity (purple), vesicle transport (light blue), cytoskeletal binding (green), protein kinases (orange), components of the striatin-interacting phosphatase and kinase (STRIPAK) protein complex (green), and cell junctions (yellow). Edge width reflects protein spectral counts, a semiquantitative measure of association strength. For simplicity, interactions mapping to wild-type and kinase-dead mutants of MST1 and MST2 are combined and represented by a single MST1/2 node. Interactions of particular interest are highlighted in red. (B) Spectral counts for selected interacting proteins identified with the indicated bait proteins. SLMAP, STRN/3/4, and SIKE1 are components of the previously described STRIPAK complex. (C) Coimmunoprecipitation (co-IP) of endogenous DLG5 and MST1/2 proteins. Western blot analysis of total (input) and immunoprecipitated protein using control IgG (IP:IgG), anti-DLG5 (IP:DLG5), or anti-MST1/2 (IP:MST1/2) antibodies. See also Supplemental Figure S1.

Conversely, co-IP with anti-MST1/2, but not negative control IgG, pulled down DLG5 from wild-type cell extracts. Thus, we conclude that endogenous DLG5 and MST1/2 proteins stably interact with each other.

Prominent activation of Hippo signaling in $\mathrm{Dlg} 5^{-/-}$mice

Since DLG5 interacts physically with MST1/2 kinases, which play pivotal roles in Hippo signaling (Mo et al.
2014), we investigated whether the Hippo pathway is affected in $D \lg 5^{-1-}$ mice. Dlg5 is required for proper brain development; therefore, we analyzed Hippo signaling in E14.5 brains (Fig. 2A). As protein levels and phosphorylation may vary between different individuals, we independently analyzed protein extracts from five wild-type and five $D l g 5^{-1-}$ embryos.

We found that the specific phosphorylation at the activation sites of MST1/2 and LATS1/2 (Yu and Guan 2013) was increased in E14.5 Dlg $5^{-/-}$brains relative to littermate controls (Fig. 2A,B). Active LATS1/2 
phosphorylates the main downstream Hippo pathway effectors YAP/TAZ, promoting their nuclear exclusion and degradation (Mo et al. 2014). Indeed, total protein levels of both YAP and TAZ were prominently decreased, and the specific phosphorylation of LATS1/2 target residue at $\mathrm{YAP}(\mathrm{S} 127)$ was substantively increased in E14.5 Dlg $5^{-/-}$brains (Fig. 2A,B). We also noted a modest decrease in total MST1/2 and LATS1/2 levels in Dlg5 ${ }^{-/}$ -, some of which may be due in part to feedback mechanisms working to maintain pathway homeostasis (Moroishi et al. 2015).

To determine whether these changes in the Hippo pathway in $D \lg 5^{-1-}$ embryos are restricted to developing brains, we also examined total liver protein extracts from E14.5 wild-type and $D \lg 5^{-/-}$embryos (Fig. 2C). Dlg5 $5^{-/-}$livers displayed a significant decrease in the total TAZ protein levels and an increase in phosphorylation of YAP(S127). Similar changes were also observed in E14.5 skin protein extracts (Supplemental Fig. S2). Taken together, these data are consistent with constitutive activation of the Hippo signaling pathway in $D \lg 5^{-/-}$mutant tissues in vivo.

To analyze whether decreased levels of YAP and TAZ proteins result in decreased expression of their transcriptional gene targets, we performed quantitative RT-PCR (qRT-PCR) analysis to examine the transcript levels of Ctgf and Cyr61, two well-known targets (Zhao et al. 2008). As predicted, we observed a significant decrease in the mRNAs encoding both Ctgf and Cyr61, but not Yap1 or Taz (Wwtr1), in E14.5 Dlg5 $5^{-/-}$brains relative to wildtype littermates (Fig. 2D). The prominent decrease in the levels of the cognate proteins, but not in the mRNA levels of YAP and TAZ, suggests activation of YAP/TAZ protein destruction mechanisms, which is consistent with up-regulation of Hippo signaling (Mo et al. 2014).

The Hippo pathway plays an important role in brain development. Conditional brain-specific deletion of Yap1 results in failure of development of the ependymal cell layer lining the brain ventricles, closure of the aqueduct, and prominent hydrocephalus (Park et al. 2016). Remarkably, $D \lg 5^{-/-}$mice also fail to develop the ependymal cell layer and show closure of the aqueduct and hydrocephalus (Nechiporuk et al. 2007). To identify potential underlying changes in the expression pattern and/or localization of Hippo pathway proteins in the developing $D \lg 5^{-/-}$brain, we performed immunostaining of coronal E15.5 brain sections using anti-YAP and anti-Sox2 (progenitor cell marker) antibodies (Fig. 2E).

As expected, Sox $2^{+}$NPCs were present at the ventricular wall of both wild-type and $D \lg 5^{-/-}$brains (Fig. 2E). YAP was prominently expressed in the cell nuclei and at the apical membrane domain facing the lumen in wild-type progenitor cells; however, it was barely detectable in $D \lg 5^{-/-}$brains (Fig. 2E), in agreement with our analysis of total brain protein extracts.
A

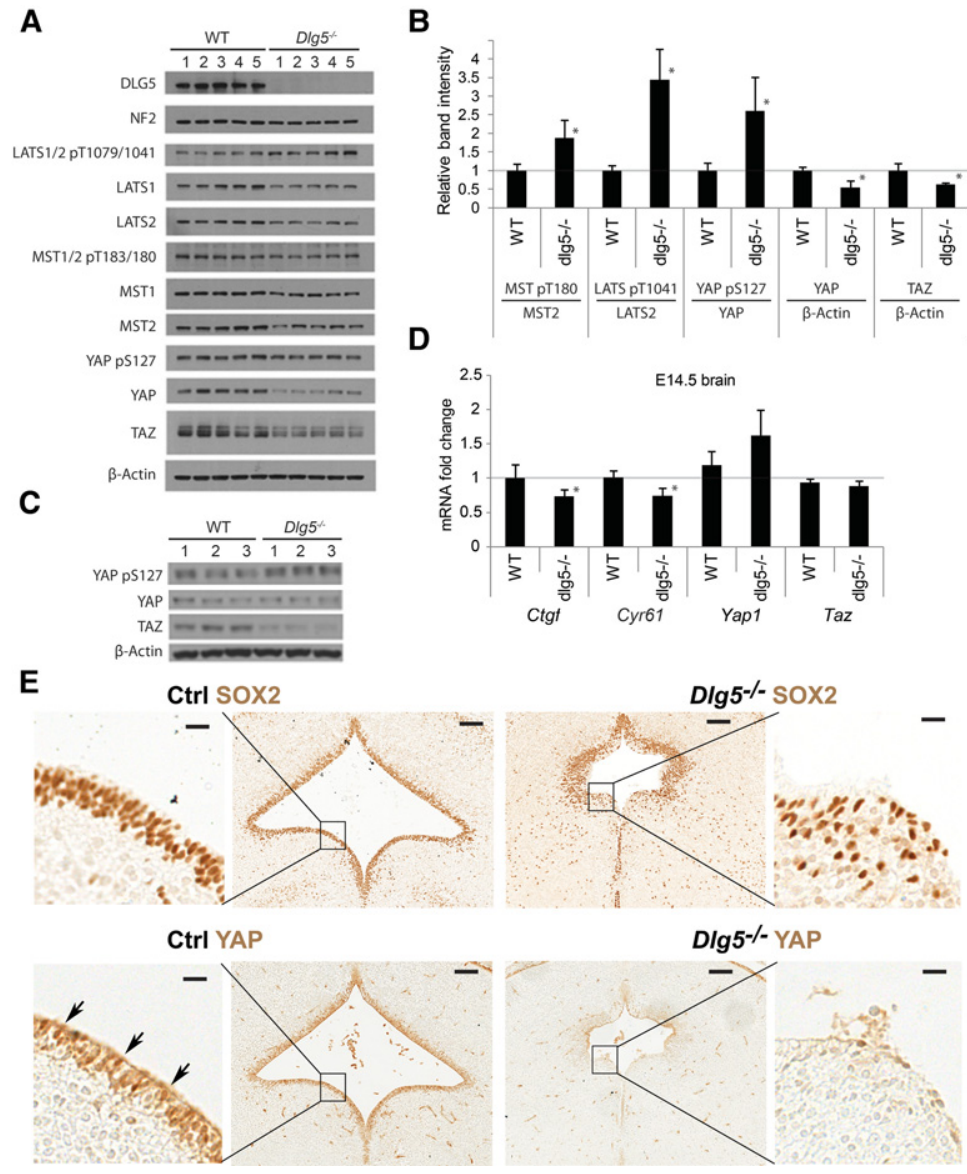

Figure 2. Activation of the Hippo signaling pathway in $D \lg 5^{-1-}$ mice in vivo. (A) Western blot analyses of total brain protein extracts from five E14.5 wild-type and five E14.5 $\mathrm{Dlg} 5^{-/}$embryos with the indicated antibodies. (B) Quantitation and normalization of phospho-specific signal to total protein or total protein to $\beta$-actin from $\left.A .{ }^{*}\right) P<0.05$, Student's $t$-test. $(C)$ Western blot analyses of total liver protein extracts from three E14.5 wild-type and three $D \lg 5^{-/-}$embryos with antibodies against YAP phospho-S127, total YAP, total TAZ, and $\beta$-actin. (D) Quantitative RT-PCR (qRT-PCR) analysis of mRNA transcript levels of Ctgf, Cyr61, Yap1, and Taz (Wwtr1) in brains from three E14.5 wild-type and three E14.5 $\mathrm{Dlg} 5^{-/}$embryos. $\left(^{*}\right) P<0.05$, Student's $t$ test. (E) Immunohistochemical staining of coronal brain sections at the level of future aqueducts from wild-type (Ctrl) and Dlg5 ${ }^{-/-}$E15.5 embryos with anti-YAP1 and anti-SOX2 (NPC, marker) antibodies. Note decreased YAP1 expression in $\mathrm{Dlg} 5^{-1-}$ NPCs. Outlined areas of the ventricular wall on low-magnification frames (middle) are shown at higher magnification (margins). Bars: $E$, middle, low-magnification images, $100 \mu \mathrm{m} ; E$, margins, higher-magnification images, $15 \mu \mathrm{m}$. See also Supplemental Figure S2. 
Conditional deletion of Mst1/2 rescues phenotypes in brain-specific Dlg5 knockout mice

Since we found that DLG5 interacts with MST1/2 and that Hippo signaling is up-regulated in $D \lg 5^{-/-}$mice, we reasoned that if DLG5 negatively regulates the Hippo pathway via MST1/2, and activation of this pathway is responsible for the developmental defects in $D \lg 5^{-1-}$ mutants, then deletion of Mst1/2 genes should rescue the phenotype of $D \lg 5^{-/-}$mice. Since knockout of $M s t 1 / 2$ results in embryonic lethality (Oh et al. 2009), we decided to address this question using a conditional brain-specific knockout approach. We generated mice with a conditional-ready $D \lg 5^{\text {flox }}$ allele using embryonic stem cell gene targeting technology, and a brain-specific knockout of $D \lg 5$ was achieved by crossing $D \lg 5^{\text {flox/flox }}$ mice with Nestin-Cre, which is expressed in central nervous system (CNS) stem/neural progenitors starting at E10.5 (Fig. 3A, B; Graus-Porta et al. 2001).

Similar to a previously reported brain phenotype in Dlg5-/- mice (Nechiporuk et al. 2007), Nestin-Cre/ $D \lg 5^{\text {flox/flox }}$ (Dlg5 conditional knockout [CKO]) mice failed to develop the ependymal cell layer, presented with a closed aqueduct, and developed severe hydrocephalus (Fig. 3C-E). Remarkably, these phenotypes were completely rescued in Nestin-Cre/DIg $5^{\text {flox/flox }} / M_{\text {st } 1 / 2^{\text {flox/flox }}}$ mice, which developed the ependymal cell layer $\left(S 100 \beta^{+}\right.$ cells), had open aqueducts, and did not develop hydrocephalus (Fig. 3C-E). Nestin-Cre/Mst1/2 flox/flox mice were similar to wild-type controls. Thus, genetic analysis in mice demonstrated requirements for Mst1/2 for the development of Dlg5-mediated phenotypes.

In addition to genetic interaction between $M s t 1 / 2$ and $D \lg 5$, we also analyzed potential genetic interactions between Dlg5 and Yap1/Taz. We reasoned that if the developmental phenotypes in $D l g 5^{-/-}$mice are due to excessive activation of Hippo signaling and subsequent inhibition of YAP and TAZ, then the phenotype of $D \lg 5^{-/-}$ animals should be aggravated upon additional loss of one or both gene copies of Yap1 or Taz (Wwtr1). Indeed, we found that deletion of a single copy of Yap1 results in lethality in $D \lg 5^{-/-} / \mathrm{Yap}^{+/-}$mice (Supplemental Fig. S3A). Similarly, while homozygous $W w t r 1^{-/-}$mice are viable and fertile, double-mutant $D \lg 5^{-/-} / W w t r 1^{-/-}$pups showed a pronounced neonatal lethal phenotype (Supplemental Fig. S3B,C). We conclude that both mammalian Yap1 and Taz (Wwtr1) show strong genetic interactions with Dlg5, supporting an important role of Dlg5 in regulating the Hippo signaling cascade.

\section{Dlg5 inhibits Hippo signaling during fly development}

The Hippo pathway was initially discovered in Drosophila (Harvey et al. 2003; Wu et al. 2003). While there are remarkable similarities between the Hippo pathways present in flies and mammals, there are also notable differences (Klezovitch and Vasioukhin 2015; Yang et al. 2015). Hence, to determine whether DLG5 function in negative regulation of Hippo signaling is evolutionarily conserved, we used RNAi-mediated knockdown to ana- lyze the signaling role of Dlg5 in flies. The Drosophila ortholog of mammalian Dlg5 is essential for embryonic development (Reilly et al. 2015). Expression of five RNAi constructs targeting different regions of dlg5 in either the Drosophila eye or the posterior wing compartment resulted in a markedly reduced size of these organs in adult flies (Fig. 4A-C).

To confirm whether expression of the $\operatorname{dlg} 5$ RNAi constructs resulted in misregulation of the Hippo pathway, we monitored Yki (Drosophila YAP/TAZ ortholog) activity using an established ex-LacZ reporter (Yang et al. 2015). Expression of $d \lg 5$ RNAi using either engrailed or decapentaplegic drivers resulted in reduced LacZ staining (Fig. 4D-G). The reduced Yki reporter activity and a corresponding reduction in compartment size in tissues expressing dlg5 RNAi strongly suggest that Drosophila dlg5 acts as a conserved negative regulator of Hippo signaling, consistent with our findings in mammalian model systems.

\section{DLG5 negatively regulates Hippo signaling in nonpolarized mouse and human cells}

As we observed an increase in Hippo signaling in $D \lg 5^{-/-}$ mice in vivo (Fig. 2), our findings suggested an important role of DLG5 in Hippo pathway regulation. However, prominent apical-basal cell polarity defects are present in $D \lg 5^{-1-}$ embryos (Nechiporuk et al. 2007), and loss of apical-basal cell polarity may indirectly impact Hippo signaling, although general loss of cell polarity usually results in inactivation of the Hippo pathway (Boggiano and Fehon 2012; Szymaniak et al. 2015; Yang et al. 2015). Therefore, to rule out an indirect effect of Dlg5 inactivation on Hippo signaling due to apical-basal cell polarity defects in $D \lg 5^{-/-}$embryos, we analyzed Hippo signaling in $D \lg 5^{-/-}$cells in two-dimensional (2D) tissue culture conditions, where both wild-type and $D \lg 5^{-1-}$ cells do not maintain apical-basal polarity. In these experiments, we decided to concentrate on primary NPCs, since these cells display a prominent phenotype in E15.5 $\mathrm{Dlg} 5^{-/-} \mathrm{em}$ bryos (Nechiporuk et al. 2007; Chang et al. 2010).

NPCs were isolated from three E12.5 wild-type and three $D \lg 5^{-/-}$brains and cultured on plastic plates coated with laminin in serum-free medium conditions that support long-term self-renewal (Sun et al. 2008). After passaging cells for $3 \mathrm{wk}$, total protein levels were analyzed by immunoblotting. Similar to our in vivo results, we observed a decrease in total YAP and TAZ protein levels and prominent activation of LATS1/2 (Fig. 5A,B). Moreover, qRT-PCR analyses of the endogenous gene targets of Hippo signaling Ctgf and Cyr61 revealed a prominent decrease in expression in $D \lg 5^{-/-}$cells (Fig. 5C), whereas the levels of Yap1 and Taz (Wwtr1) mRNAs were unchanged. Overall, these findings demonstrate that the increase in Hippo pathway signaling in $D \lg 5^{-/-}$cells is independent of defects in the maintenance of apical-basal cell polarity in $D \lg 5^{-/-}$embryos.

To determine whether, in addition to mouse cell models, DLG5 also negatively regulates the Hippo signaling pathway in human cells, we next analyzed human breast 


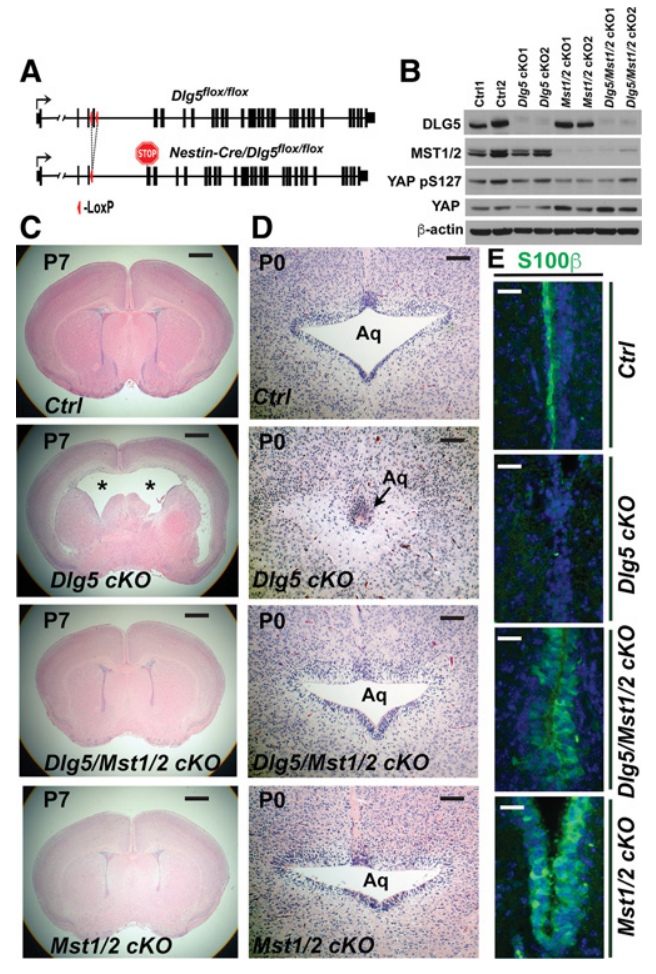

Figure 3. Conditional deletion of $M s t 1 / 2$ rescues phenotypes in brain-specific Dlg5 knockout mice. (A) Schematic representation of conditional $\left(D \lg 5^{\text {flox } / f l o x}\right)$ and Cre-recombined (Dlg5 conditional knockout [cKO]) Dlg5 alleles. (B) Western blot analyses of total brain protein extracts from newborn control wild-type, $D \lg 5 \mathrm{cKO}$, $M s t 1 / 2 \mathrm{cKO}$, and triple $D \lg 5 / M s t 1 / 2$ cKO pups with the indicated antibodies. $(C, D)$ Haematoxylin and eosin staining of coronal sections from postnatal day $7(\mathrm{P} 7)(C)$ and $\mathrm{P} 0(D)$ wild-type $(\mathrm{Ctrl}, n=$ 10 for P7; $n=7$ for P0), Nestin-Cre/Dlg5 flox/flox $(D \lg 5 \mathrm{cKO}, n=5$ for P7; $n=3$ for P0), Nestin-Cre/Dlg $5^{\text {flox } / \text { flox }} / M s t 1 / 2^{\text {flox/flox }}$ (Dlg5/Mst1/2 cKO, $n=10$ for P7; $n=5$ for P0), and Nestin-Crel Mst1/2 flox/flox (Mst1/2 cKO, $n=5$ for P7; $n=4$ for P0) brains. Note the severe dilation of lateral ventricles (asterisks) and closure of the aqueduct ( $\mathrm{Aq}$; arrow) in Dlg5 cKO brains. (E) Immunofluorescent staining of lateral ventricles from $\mathrm{P} 7$ wild-type (Ctrl), $D \lg 5$ cKO, Dlg5/Mst1/2 cKO, and Mst1/2 cKO mice with antiS100 $\beta$ (ependymal cell marker) antibodies (green). $n=3$ for each genotype. DAPI was used as a nuclear counterstain (blue). Bars: $C, 0.7 \mathrm{~mm} ; D, 0.1 \mathrm{~mm} ; E, 40 \mu \mathrm{m}$. See also Supplemental Figure S3.

cancer (MDA-MB-231) and liver cancer (HepG2) cell lines after transient knockdown of endogenous DLG5. Perturbation of DLG5 in MDA-MB-231 resulted in an increase in YAP phosphorylation, which was evident by a decrease in the levels of fast-migrating (nonphosphorylated) YAP as revealed by electrophoresis on PhosTag gels (Fig. 5D). In addition, immunofluorescent staining of YAP in MDAMB-231 cells revealed that DLG5 knockdown resulted in a pronounced loss of the predominately nuclear localization of YAP (Fig. 5E).

PhosTag gel electrophoresis revealed that, unlike in MDA-MB-231 cells, the majority of YAP was already constitutively hyperphosphorylated in HepG2 cells (Fig. 5D).
Rather, instead of an increase in YAP phosphorylation, we observed that knockdown of $D L G 5$ in HepG2 resulted in a specific decrease in total YAP protein levels (Fig. 5D). In addition, we also observed a corresponding decrease in the expression levels of endogenous Hippo pathway transcriptional targets (CTGF, CYR61, and ANKRD1) in both $D L G 5$ knockdown cell lines, whereas YAP1 and TAZ transcript levels remained unchanged (Fig. 5F). The modest decrease in total MST1 and LATS1 levels in both knockdowns may reflect other feedback mechanisms working to maintain pathway homeostasis.

Overall, the phenotypes that we observed were consistent with hyperactivation of Hippo signaling upon DLG5 knockdown. Therefore, we conclude that DLG5 negatively regulates Hippo signaling in both human and mouse cells.

\section{Hippo signaling is negatively impacted by gain of function of DLG5}

While we determined that Dlg5 loss of function results in increased activity of the Hippo pathway in various model systems, it was still possible that DLG5 is not a genuine member of the Hippo signaling pathway and that inactivation of Dlg5 influences Hippo signaling indirectly. We reasoned that a member of the Hippo pathway should be able to affect signaling not only upon loss of function but also in gain-of-function scenarios. We found that overexpression of DLG5 in HEK293T cells did not affect the total levels of exogenous MST2 and LATS1 but resulted in a prominent increase in the levels of exogenous YAP and TAZ (Supplemental Fig. S4A,B). These DLG5-mediated changes were especially pronounced when both MST2 and LATS1 were overexpressed together in the same cells (Supplemental Fig. S4A,B, brackets). Thus, we concluded that DLG5 positively regulates YAP and TAZ protein levels in not only loss-of-function experiments but also gainof-function experiments.

To examine how DLG5 might be inhibiting the Hippo pathway, we performed co-IP experiments using epitopetagged MST2, LATS1, SAV1, and DLG5 constructs. As expected, MST2 pull-down also enriched for LATS1; however, overexpression of DLG5 decreased the interaction between MST2 and LATS1 but not between MST2 and SAV1, indicating that DLG5 specifically interferes with the binding between MST2 and LATS1 (Fig. 6A).

To confirm whether DLG5 gain of function impacts the downstream transcriptional activity of the Hippo pathway, we performed luciferase readout assays using a reporter containing a CTGF promoter, which is directly regulated by Hippo signaling (Lai et al. 2011). As expected, overexpression of MST2 and LATS1 negatively impacted TAZmediated activation of the CTGF reporter (Supplemental Fig. S4C), whereas overexpression of DLG5 significantly attenuated Hippo signaling, as evidenced by a marked increase in the activity of the CTGF reporter (Supplemental Fig. S4C), demonstrating a negative role of DLG5 in regulating the Hippo pathway in these gain-of-function experiments. 

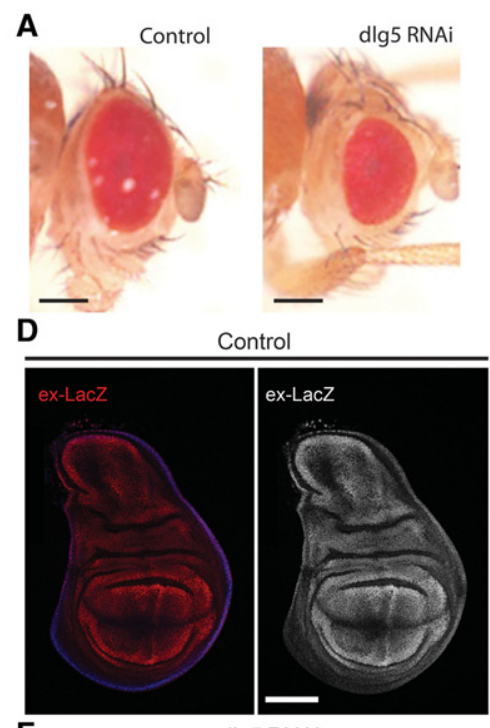

$\mathbf{F}$

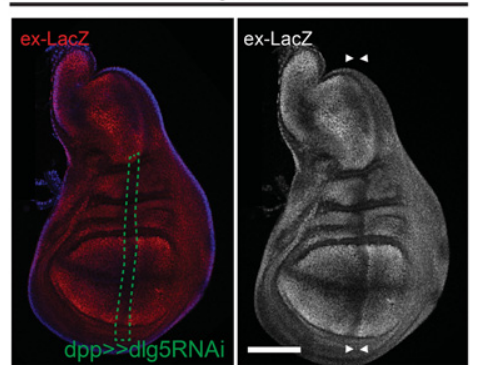

B
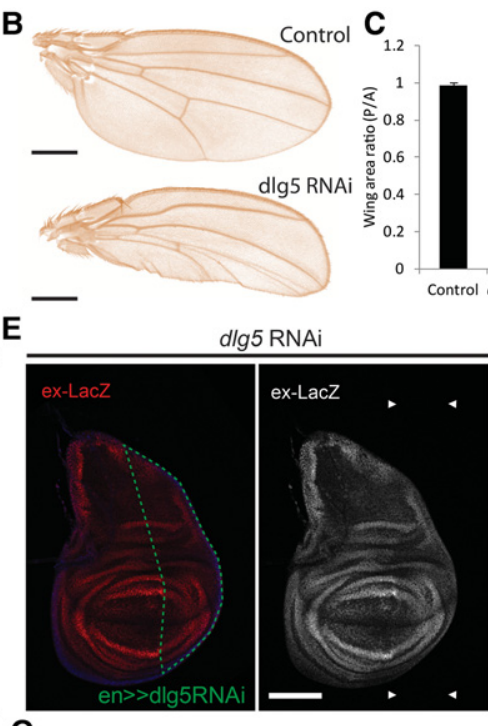

G

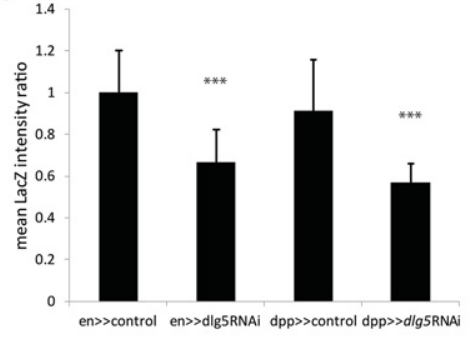

Figure 4. Activation of Hippo signaling in dlg5 knockdown flies. (A) Expression of dlg5 RNAi in developing eyes results in growth defects. Representative images of Drosophila heads from driver controls and ey-Gal4-driven dlg5 RNAi flies. Bar, $200 \mu \mathrm{m}$. (B) Expression of dlg5 RNAi in posterior wing compartment results in reduced size. Representative images of wings from driver controls and en-Gal4-driven dlg5 RNAi flies. Bar, $200 \mu \mathrm{m} .(C)$ Posterior $(\mathrm{P})$ to anterior $(\mathrm{A})$ wing area ratio for control and en $>>d l g 5$ RNAi wings. $\left({ }^{* *}\right) P<0.001$, Student's unpaired $t$-test. $(D-F)$ Yki reporter exlacZ (red in the left panel and grayscale in the right panel) wing disc staining in control $(D)$ or dlg5 RNAi driven by either engrailed (en-Gal4) $(E)$ or decapentaplegic (dpp-Gal4) (F) Gal4. The respective expression areas are outlined in green. DAPI staining is shown in blue. Bar, $100 \mu \mathrm{m}$. (G) Reporter intensity ratio for mutant tissue and adjacent nonmutant tissue areas. Chosen areas are representative of the heterogeneity of the tissue. $\left(^{* * *}\right)$ $P<0.001$, Student's unpaired $t$-test.
The third PDZ domain of DLG5 binds MST1/2 but is not sufficient for Hippo inhibition

DLG5 is a large multidomain protein. To determine which domains of DLG5 are responsible for interaction with MST1/2, we performed co-IP experiments using a panel of recombinant DLG5 fragments and internal deletion constructs expressed in HEK293T cells. We found that the third PDZ domain (amino acid position 13491436) of DLG5 was sufficient for strong binding to MST1/2 (Fig. 6B,C; Supplemental Fig. S5A).

To test whether the interaction between PDZ3 of DLG5 and MST1/2 was direct or instead mediated through another adaptor protein (for example, SAV1), we performed in vitro pull-down assays with recombinant GST-PDZ domains and stringently purified MST1/2 proteins. We found that GST-PDZ3 (of DLG5), but not PDZ1, PDZ2, or PDZ4, was able to bind MST1/2, strongly suggesting that the interaction between DLG5 and MST1/2 proteins is direct (Fig. 6D; Supplemental Fig. S5B).

We also tested the activity of various DLG5 constructs using a TEAD luciferase reporter. Full-length DLG5 strongly activated the reporter, but deletion of PDZ domains 3 and 4 greatly reduced reporter activation (Fig. $6 \mathrm{E})$. Intriguingly, a construct consisting of only PDZ3 of DLG5, which is sufficient for binding to MST1/2, failed to stimulate transcription (Fig. 6E). These results indicate that DLG5 needs to associate with MST1/2 for Hippo pathway inhibition; however, binding of the PDZ3 domain alone to MST1/2 is not sufficient for this regulation. Thus, we hypothesized that DLG5 might act as a molecular scaffold in which the other protein domains of DLG5 play an important role, perhaps by recruiting additional regulatory proteins.

\section{MARK3 inhibits the Hippo signaling pathway}

Our initial AP/MS screens revealed a physical interaction between DLG5 and MARK3 and, to a lesser extent, the paralogous proteins MARK2 and MARK1 (Fig. 1A; Supplemental Table S1). Mammalian MARK1-4 are orthologs of Drosophila Par-1, which plays a critical role in apicalbasal cell polarity. To confirm interaction between endogenous DLG5 and MARK3, we performed co-IP experiments between these proteins in murine primary NPCs. Immunoprecipitation with anti-DLG5, but not negative control IgG antibodies, pulled down MARK3 protein (Fig. 7A). Conversely, co-IP with anti-MARK3, but not negative control IgG, pulled down DLG5 (Fig. 7A). Thus, we conclude that endogenous DLG5 and MARK3 proteins stably interact with each other. Moreover, our AP/MS data indicated that kinase-dead MST2 interacts with both DLG5 and MARK3, suggesting the possibility of DLG5-MST1/2-MARK3-containing complexes (Fig. 1B). 


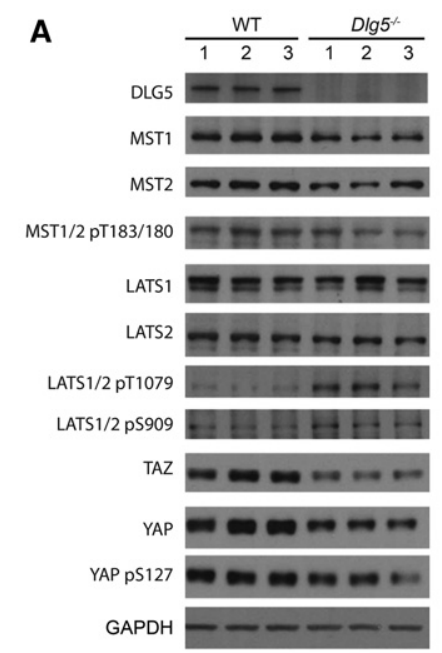

B

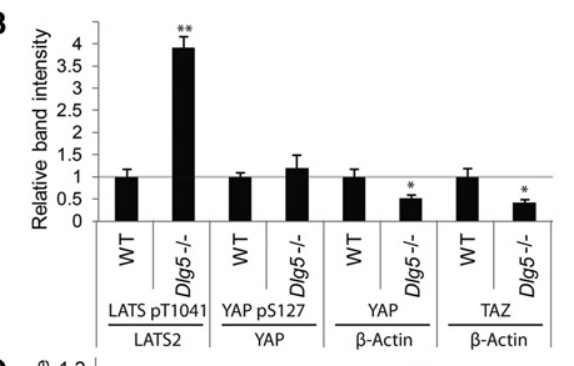

C
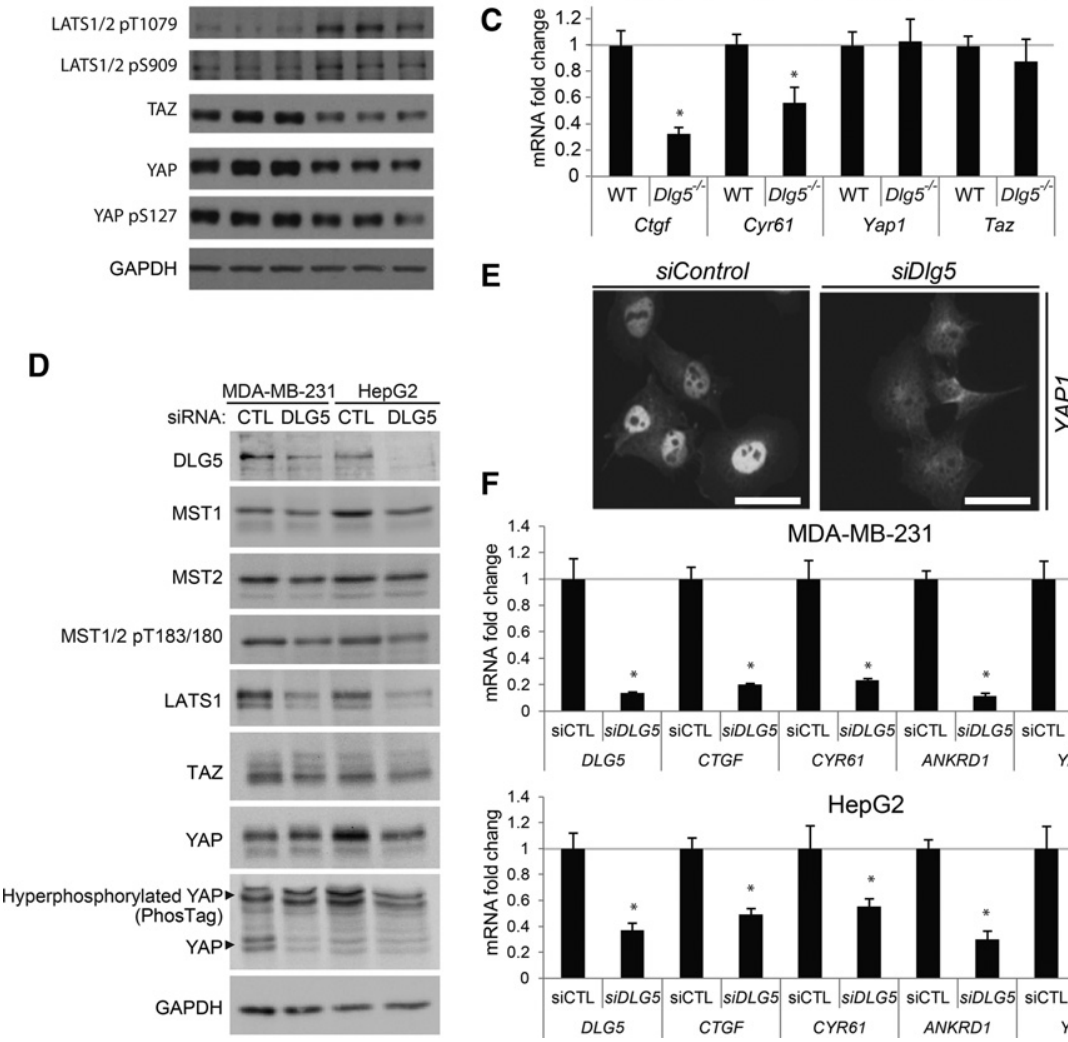

$E$
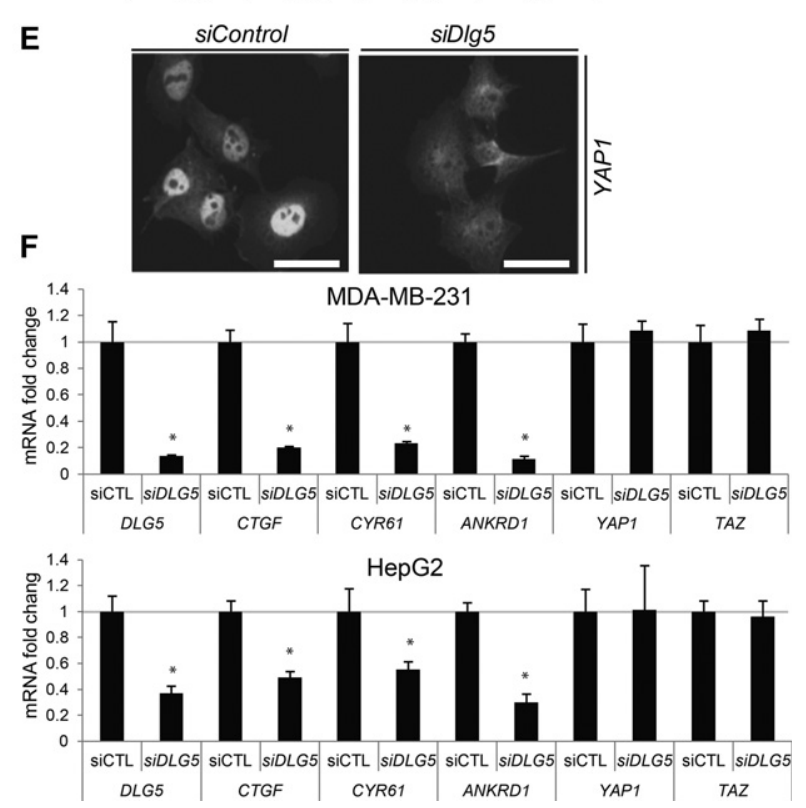

Figure 5. Dlg5 negatively regulates Hippo signaling in nonpolarized cells in culture. $(A)$ Western blot analyses of total protein extracts from primary NPC cultures established from three E12.5 wild-type and three $D \lg 5^{-/}$embryos and blotted with indicated antibodies. (B) Quantitation and normalization of the phospho-specific signal to total protein or total protein to $\beta$-actin from $A$. $\left(^{*}\right) P<0.05$; $\left({ }^{* *}\right) P<$ 0.01, Student's $t$-test. (C) qRT-PCR analyses of mRNA encoding Ctgf, Cyr61, Yap1, and Taz (Wwtr1) in NPC cultures in $A$. $\left({ }^{*}\right) P<0.01$, Student's $t$-test. $(D)$ Western blot analyses of total protein from MDA-MB-231 and HepG2 cells transfected with siControl (CTRL) or siDLG5 oligos and analyzed with the indicated antibodies. $(E)$ Immunofluorescent staining of endogenous YAP in MDA-MB-231 cells transfected with siControl or siDLG5 oligos. Bar, $50 \mu \mathrm{m}$. $(F)$ qRT-PCR analysis of DLG5, CTGF, CYR61, ANKRD1, YAP1, and TAZ (WWTR1) transcript levels in MDA-MB-231 and HepG2 cells transfected with siControl (CTL) or siDLG5 oligos. $\left({ }^{*}\right) P<0.01$, Student's $t$-test.

In Drosophila, MARK ortholog Par-1 negatively regulates the activity of hpo kinase, and overexpression of human MARK4 inhibits the Hippo pathway in HEK293T cells (Huang et al. 2013). To examine the role of MARK3 in Hippo pathway regulation, we overexpressed MARK3 in HEK293T cells. MARK3 expression resulted in prominent activation of a TEAD luciferase reporter (Fig. 7B). Furthermore, coexpression of DLG5 and MARK3 had a synergistic effect on reporter activation (Fig. 7B). Taken together, these results are consistent with a putative role of DLG5 in acting as a molecular scaffold to influence MST1/2 activity.
DLG5 promotes MST1/2 association with MARK3 and inhibition of MST1/2-mediated phosphorylation of LATS1

To determine the domain of DLG5 mediating the interaction with MARK3, we performed co-IP experiments between MARK3 and various fragments of DLG5. Fulllength DLG5 and the N-terminal construct, but not any other domain, bound MARK3 (Fig. 7C; Supplemental Fig. S6A).

Since the N-terminal DLG5 construct contains three domains (CARD, DUF, and coiled-coil), we expressed 


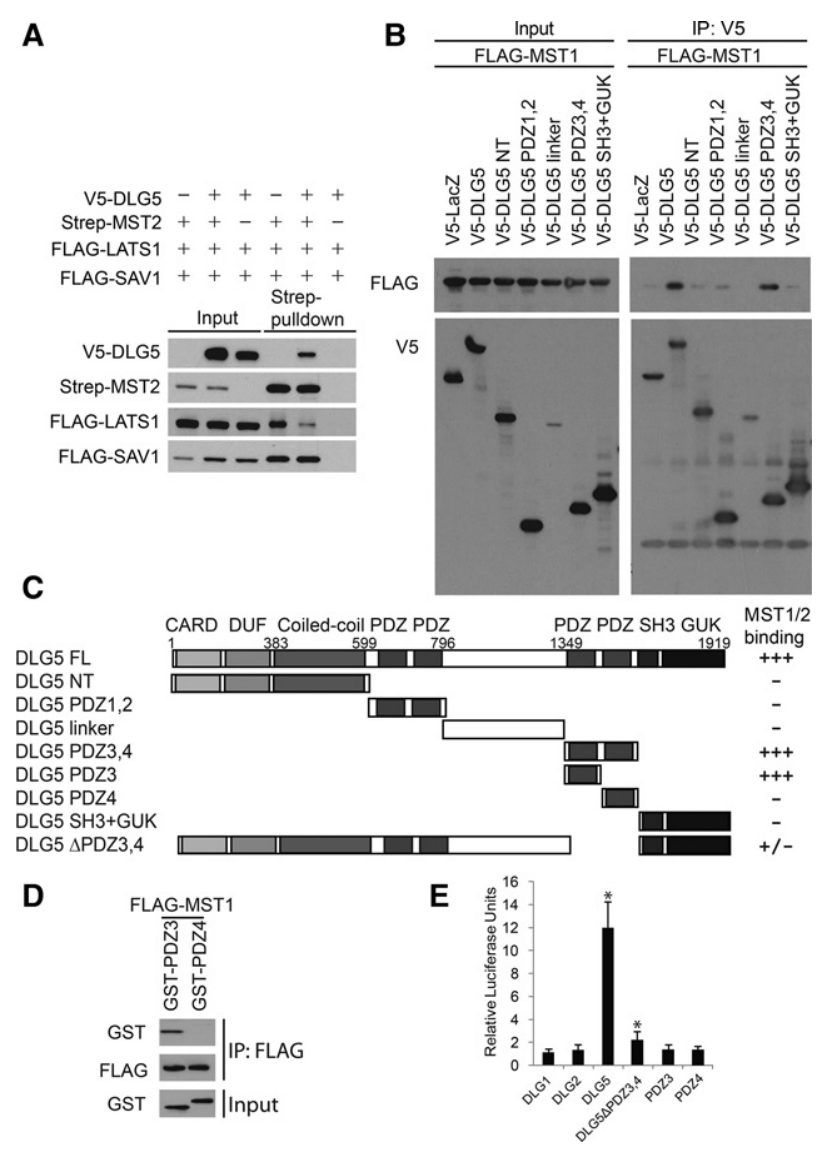

Figure 6. DLG5 binds to MST1/2 and interferes with MST2LATS1 interaction. (A) Western blot analyses of total protein (input) and affinity pull-downs using Strep-Tactin-Sepharose from HEK293FT cells transfected with V5-DLG5, Strep-MST2, FlagLATS1, and Flag-SAV1 expression constructs, as indicated. Note that DLG5 inhibits the binding of MST2 to LATS1. (B) PDZ3-4 domains of DLG5 bind MST1. Western blot analysis of total and anti-V5 immunoprecipitated (IP:V5) proteins from HEK293 cells expressing Flag-MST1 and the indicated V5-tagged fragments of DLG5. (C) Diagram showing DLG5 fragment/deletion constructs used to identify the MST1/2-binding domain. Symbols indicate the relative strength of binding summarized from $D$ and Supplemental Figure S5. (D) PDZ3 of DLG5 binds directly to MST1. Western blot analyses of input and anti-FlagSepharose pulled-down proteins with anti-Flag and anti-GST antibodies. GST fusions with PDZ3 and PDZ4 domains of DLG5 were purified from bacteria. Flag-MST1 was purified from HEK293FT cells. (E) PDZ3/4 domains of DLG5 are necessary but not sufficient for YAP/TAZ reporter activation. Luciferase transcriptional activity assay using a promoter containing multimerized TEAD-binding sites. Cells were cotransfected with the reporter, control, and indicated expression constructs. Error bars represent means \pm SD. Student's $t$-test. $n=3$. See also Supplemental Figures S4 and S5.

and purified GST fusions of these domains from bacteria. We found that only the coiled-coil domain of DLG5 interacted with tagged MARK3, purified from HEK293 cells, indicating that DLG5 binds to MARK3 via its coiled-coil domain (Supplemental Fig. S6B) and that this interaction is likely direct.
Since DLG5 binds to both MST1/2 and MARK3 via separate domains, we hypothesized that DLG5 promotes association between MST1/2 and MARK3. Indeed, co-IP experiments revealed a pronounced dose-dependent increase in the interaction between MST1/2 and MARK3 in cells coexpressing DLG5 (Fig. 7D; Supplemental Fig. S6C,D).

Drosophila Par-1 negatively regulates the activity of Hpo kinase by promoting inhibitory hyperphosphorylation of Hpo (Huang et al. 2013). This hyperphosphorylation is distinct from specific phosphorylation of $\mathrm{Hpo} /$ MST1/2 at the kinase activation loop, which, in contrast, marks kinase activation. In mammals, the Par-1 homolog MARK4 promotes YAP/TAZ activity and induces hyperphosphorylation of MST2, although whether this hyperphosphorylation regulates MST2 activity is not yet known (Huang et al. 2013).

Using PhosTag gel electrophoresis, we examined whether either MARK3 or DLG5 promotes hyperphosphorylation of MST2. Indeed, MARK3 overexpression induced hyperphosphorylation of MST2, which was further enhanced by coexpression of full-length DLG5 (Fig. 7E). Consistent with a putative scaffolding function of DLG5 in linking MARK3 and MST1/2, the PDZ3 domain of DLG5 alone, which binds to MST2 but not MARK3, was insufficient for promoting MARK3-mediated hyperphosphorylation of MST2 (Fig. 7E).

To directly access whether MARK3 and DLG5 can influence MST1/2 kinase activity in the Hippo signaling pathway, we performed in vitro kinase assays with purified MST2, MARK3, and DLG5 proteins. Recombinant GST-LATS1 was used as a substrate, and MST2 activity was monitored by Western blotting with anti-phospho[Thr1079]-LATS antibodies. Strikingly, while DLG5 and MARK3 separately had a modest negative impact, the combination of DLG5 and MARK3 prominently and synergistically inhibited MST2-specific phosphorylation of LATS1 (Fig. 7F). Thus, DLG5 and MARK3 cooperate to directly inhibit the kinase activity of MST1/2 toward LATS1 and thus act as negative regulators of the Hippo pathway.

\section{Discussion}

We determined previously that mammalian DLG5 functions as an apical-basal cell polarity protein (Nechiporuk et al. 2007, 2013; Chang et al. 2010). We also noticed that $D \lg 5^{-1-}$ mice are smaller than their wild-type littermates, and immunocytological analysis of their brains revealed a prominent defect in brain development (Nechiporuk et al. 2007; Chang et al. 2010). However, the molecular mechanisms responsible for these phenotypes remained unknown. We report here that DLG5 binds to MST1/2 and functions as an important, evolutionarily conserved negative regulator of MST1/2 and the entire Hippo signaling pathway (Fig. 7G). We found that Hippo signaling is hyperactive in $\mathrm{Dlg}^{--}$- embryos and that genetic deletion of Mst1/2 fully rescues the $D \lg 5^{-/-}$mutant phenotypes. This is not a secondary effect due to the role of 
DLG5 in the maintenance of apical-basal cell polarity because the negative impact of DLG5 on Hippo signaling is maintained in nonpolarized 2D cultures of NPCs. Moreover, we demonstrate that DLG5 inhibits Hippo signaling in loss-of-function assays and gain-of-function experiments.

The Hippo pathway is uniquely sensitive to the cellular microenvironment, providing a direct connection between extracellular matrix conditions, cell density, and apical-basal polarity to the transcriptional regulation of genes playing a pivotal role in cellular self-renewal and differentiation (Zhao et al. 2007; Dupont et al. 2011; Enomoto and Igaki 2011; Genevet and Tapon 2011). Molecular mechanisms responsible for these connections are only beginning to be elucidated. We found that the apical-basal polarity protein DLG5 binds directly to MST1/ 2 through its third PDZ domain and negatively regulates the activity of the Hippo pathway. This provides a novel direct mechanistic link between cell polarity proteins and core kinases of the Hippo signaling pathway.

Using multiple $\mathrm{AP} / \mathrm{MS}$ baits, we further characterized a network of relevant physical interactions and identified a novel protein complex that constitutes a unique mode of regulation of MST1/2, which is distinct from the RASSFs and STRIPAK assemblies reported previously (Couzens et al. 2013). We found that DLG5 interacts directly with MST1/2 and inhibits the interaction of MST2 with LATS1. In addition, DLG5 functions as a scaffold protein that uses different domains to mediate its interaction with both MARK3 and MST1/2, bringing them together to promote MARK3-dependent hyperphosphorylation and inactivation of MST1/2 kinase activity toward LATS1. Precisely which MST1/2 sites are phosphorylated and whether or how these phosphorylation events might inhibit MST2 function are matters of ongoing investigation.

Our findings reveal DLG5 as a novel mechanistic link between cell polarity proteins and the Hippo signaling pathway. In addition to DLG5, other apical-basal polarity proteins have been implicated in regulation of the Hippo signaling pathway. Interestingly, unlike DLG5, these proteins are positive regulators of the Hippo signaling pathway. The apical membrane domain determinant Crumbs3 promotes Hippo signaling by facilitating the interaction between LATS1/2 and YAP (Szymaniak et al.

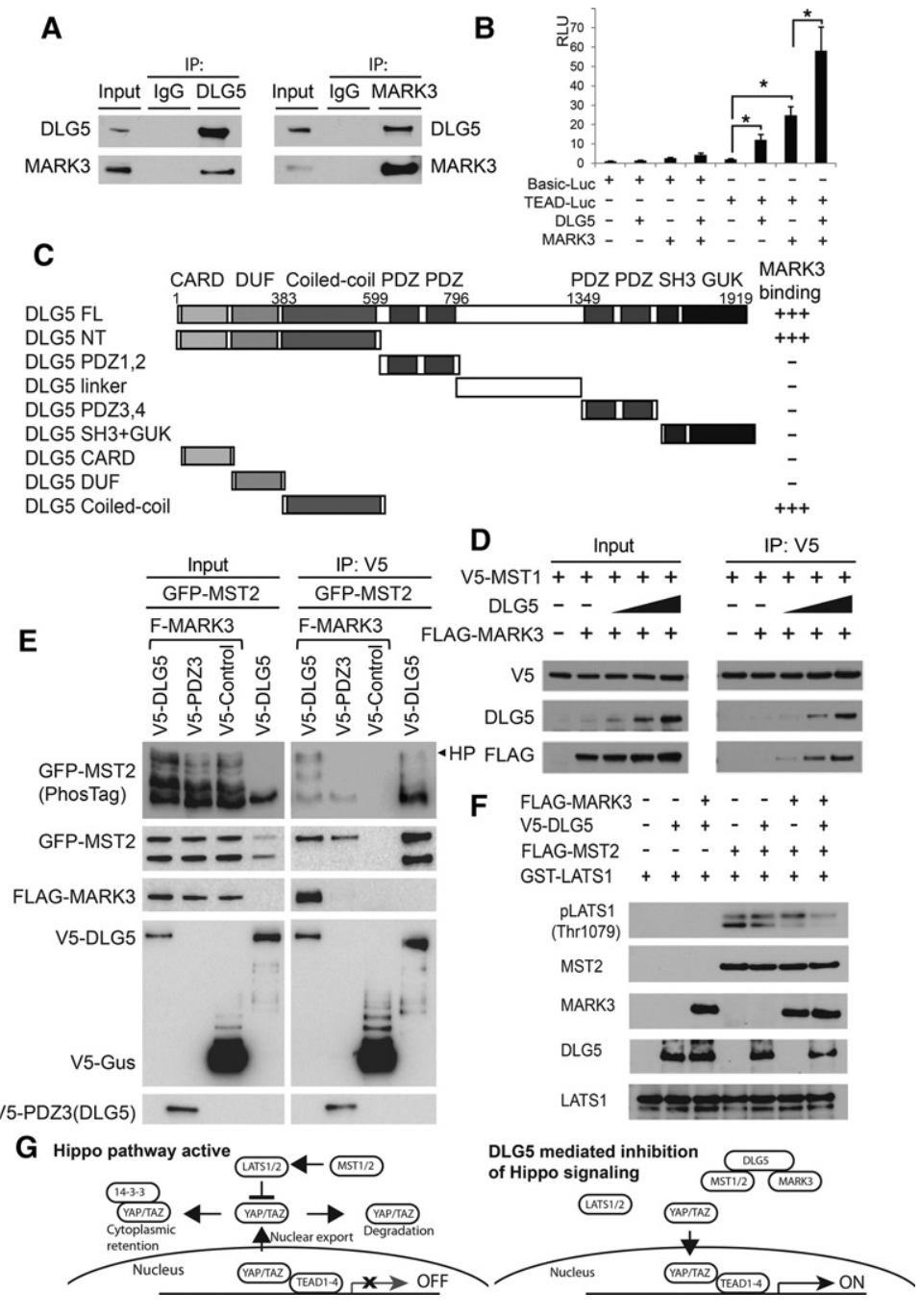

Figure 7. MARK3 is a negative regulator of Hippo signaling recruited to MST1/2 by DLG5. (A) Co-IP of endogenous DLG5 and MARK3 proteins. Western blot analysis of total (input) and immunoprecipitated using control IgG (IP:IgG), anti-DLG5 (IP:Dlg5), or anti-MARK3 (IP: MARK3) antibodies. (B) Luciferase transcriptional activity assay using empty control pGL3(Basic-Luc) or 8xTEAD reporter (TEAD-Luc) in HEK293FT cells transfected with DLG5 and/or MARK3 expression constructs, as indicated. Error bars represent means \pm SD. Student's $t$-test. $n=3$. (C) Diagram showing DLG5 fragment/deletion constructs used to identify the MARK3-binding domain. Symbols indicate binding summarized from $D$ and Supplemental Fig. S6. (D) DLG5 promotes interaction between MST1 and MARK3. Western blot analyses of total (input) and immunoprecipitated with anti-V5 antibody (IP:V5) proteins from HEK293T cells transiently transfected with Flag-MARK3, V5-MST1, and DLG5 expression constructs, as indicated. (E) Western blot analysis of total (input) and immunoprecipitated with anti-V5antibodies proteins from HEK293T cells transfected with the indicated expression constructs. Proteins were separated using either PhosTag or regular SDS-PAGE. (HP) Hyperphosphorylated. $(F)$ In vitro kinase assay with purified MST2, MARK3, DLG5, and GST-LATS proteins. Proteins were incubated in kinase buffer and then analyzed by Western blotting with the indicated antibodies. MST activity was determined by blotting with anti-phospho-LATS antibody. (G) Model of DLG5 function in Hippo signaling pathway. See also Supplemental Figure S6. 
2015). Similarly, a pivotal basolateral membrane domain determinant Scribble is a prominent positive regulator of Hippo signaling that also functions by facilitating interaction between TAZ and core Hippo kinases MST and LATS (Cordenonsi et al. 2011).

In Drosophila, Par-1, a pivotal basolateral membrane domain determinant and an ortholog of mammalian MARK proteins, acts as a negative regulator of the Hippo pathway and functions by inducing phosphorylation of Hpo and attenuating its protein kinase function (Huang et al. 2013). The role of mammalian MARK proteins in the regulation of the Hippo pathway is somewhat unclear. RNAi knockdown experiments in HEK293T cells suggested a positive role of MARK1, MARK2, and MARK4 in the regulation of the Hippo pathway downstream from LKB1 by promoting SCRIB association with MST1/ 2 and LATS1/2 and consequent Hippo pathway activation (Mohseni et al. 2014). In contrast, gain-of-function experiments with MARK1 and MARK4 in HEK293T cells uncovered a negative role of these MARK proteins in Hippo pathway regulation (Huang et al. 2013).

Our data support a negative role of MARK3 in the regulation of MST1/2 and the Hippo pathway; however, it is likely that the exact function of the same polarity proteins in regulation of the pathway may be rather complex and contextual, especially if these proteins impact several different downstream pathways that can both attenuate and increase LATS1/2-dependent phosphorylation of YAP. Furthermore, given DLG5's clear role in the inhibition of the Hippo pathway, it is surprising to see loss of DLG5 expression in prostate and bladder cancers, where it results in activation of cell invasion and metastasis (Tomiyama et al. 2015; Zhou et al. 2015). Considering our findings, $D L G 5$ may potentially play a protumorigenic rather than tumor-suppressive function. Interestingly, our analysis of the Cancer Genome Atlas (TCGA) data revealed that DLG5 is overexpressed in $\sim 5 \%$ of human clear cell carcinomas and that there is a highly significant $(P=$ 0.006 ) correlation between DLG5 overexpression and tumor recurrence (Supplemental Fig. S7). This correlative evidence supports a potential pro-oncogenic role of DLG5.

Loss of Dlg5 in mice results in multiple phenotypes, including small size, hydrocephalus, kidney cysts, and defective lung branching morphogenesis, causing an emphysema-like phenotype (Nechiporuk et al. 2007, 2013). The functional connection between DLG5 and Hippo signaling identified by our study raises an important question of whether all or some of the $D \lg 5^{-1-}$ phenotypes are caused by down-regulation of TAZ and YAP function. In this regard, there are remarkable similarities between the phenotypes of $\mathrm{Dlg} 5^{-/-}$and $\mathrm{Taz}^{-/-}$mice. Similar to Dlg5 $5^{-/-}$animals, $\mathrm{Taz}^{-/-}$mice display small size and develop kidney cysts and an emphysema-like phenotype (Hossain et al. 2007; Tian et al. 2007). While Yap1 ${ }^{-/-}$homozygous-null mice are embryonic-lethal, conditional brain-specific deletion of Yap1 results in brain phenotypes that are almost identical to brain phenotypes in Dlg5 ${ }^{-/-}$mice (Park et al. 2016; Nechiporuk et al. 2007). Moreover, we found that brain-specific deletion of Mst1/ 2 rescues $D \lg 5^{-/-}$brain phenotypes. Therefore, it is likely that all of the developmental defects found in DIg5 $5^{-/-}$ mice can be explained by down-regulation of YAP and TAZ signaling.

Our newly identified connection between DLG5 and the Hippo pathway may impact many aspects of DLG5 and Hippo signaling functions. It is still unclear which cellular and developmental processes depend on DLG5-mediated regulation of $\mathrm{YAP} / \mathrm{TAZ}$ activity and how this function of DLG5 itself is regulated. Genetic polymorphism in human DLG5 has been linked to Crohn's and inflammatory bowel disease (Friedrichs and Stoll 2006), although no mechanism for this link has been identified. It will be interesting to determine whether perturbations in DLG5-mediated regulation of Hippo signaling are responsible for the connection between intestinal inflammation and DLG5.

\section{Materials and methods}

\section{AP/MS}

HEK293T cell lines stably expressing epitope (VA)-tagged proteins were generated essentially as described (Mak et al. 2010). Affinity purification was performed as described (Kwan and Emili 2016); briefly, soluble protein lysates were generated by lysing two 15$\mathrm{cm}$ plates of cells in TBS buffer (30 mM Tris- $\mathrm{HCl}$ at $\mathrm{pH} 7.5,150$ $\mathrm{mM} \mathrm{NaCl}$ ) containing $0.5 \%$ Nonidet $\mathrm{P} 40$ and protease and phosphatase inhibitors (Complete, Roche). After incubation for 10 min on ice and clarification by centrifugation, lysates were incubated with anti-Flag M2 antibody bound to Protein G Dynabeads for $2 \mathrm{~h}$ at $4^{\circ} \mathrm{C}$. Beads were washed three times, resuspended in 50 $\mathrm{mM} \mathrm{NH} \mathrm{HCO}_{3}$, and incubated with sequencing-grade trypsin (Promega) overnight at $37^{\circ} \mathrm{C}$. Formic acid ( $2 \%$ final concentration) was added to terminate digestion, and the samples were desalted using C-18 cartridges (10-200 $\mu \mathrm{L}$ of NuTip; Glygen Corp.).

Digested peptides were resolved on a microcolumn (120 $\mathrm{mm} \times$ $75 \mu \mathrm{m})$ packed with $100 \mathrm{~mm}$ of $3-\mu \mathrm{m}$ Luna C18 stationary phase (Phenomenex) using an organic gradient of $98 \%$ buffer A $15 \%$ acetonitrile, $0.1 \%$ formic acid) to $90 \%$ buffer B $(95 \%$ acetonitrile $0.1 \%$ formic acid) over $45 \mathrm{~min}$ at a flow rate of $300 \mathrm{~nL} / \mathrm{min}$. Eluting peptides were electrosprayed directly into an Orbitrap Velos mass spectrometer (ThermoFisher Scientific), and ions with a charge state of +2 or +3 were subjected to data-dependent collision-induced dissociation. Spectra were searched using Sequest version 2.7 (Eng et al. 1994) against human (UniProtKB/SwissProt) protein sequence Fasta file. High confidence matches within $20 \mathrm{ppm}$ accurate mass were filtered using StatQuest to a false discovery rate of $<1 \%$ (Kislinger et al. 2003).

To reduce carryover artifacts, sample run order was randomized and interspersed with BSA digests, while spectral counts of peptides identified in preceding BSA digest controls were subtracted from subsequent runs. High confidence interacting proteins were scored using the compPASS algorithm (Sowa et al. 2009) and visualized using Cytoscape (Shannon et al. 2003).

\section{Mouse strains and genotyping}

Mice with a conditional Dlg5 allele containing exon 4 flanked by LoxP sequences were generated using conventional embryonic stem cell gene targeting technology and were maintained on a C57BL/6J genetic background. PCR with oligos Dlg5-CK2 (5'CTGGGGCCTAGTACAACAGG-3') and Dlg5-CK1 (5'-TCTGG CTTGGGGCTTTACTAWAS-3') was used for genotyping (wild- 
type allele, 150 base pairs [bp]; floxed allele, 320 bp). CNS-specific knockouts of Dlg5 (DIg5 cKO) were obtained by breeding mice carrying a conditional Dlg5 allele with Nestin-Cre transgenic mice (Graus-Porta et al. 2001). To generate mice with CNSspecific deletion of Mst1/2, we crossed Nestin-Cre ${ }^{+/-}$mice with Stk4tm1.1Rjo Stk3tm1.1Rjo/I mice (Jackson Laboratories, 017635). $\mathrm{Taz}^{-/+}$mice were purchased from Jackson Laboratories (011120). Yap1 ${ }^{-/+}$mice were generated by germline recombination of the Yap1 flox allele (Zhang et al. 2010) using MORE-Cre (Tallquist and Soriano 2000) mice. Both male and female mice were analyzed, and no differences were observed between the sexes. All experiments with mice were approved by the Fred Hutchinson Cancer Center Institutional Animal Care and Use Committee.

\section{Cell culture and transient transfection}

Primary NPCs were isolated from E12.5 mouse brains. The NPCs were grown on laminin-coated plates in neural stem cell growth medium (100 mL of NS-A basal medium [Stem Cell Technologies, 05750], $100 \mathrm{~mL}$ of DMEM-F12 medium [Invitrogen, 12634010], $2 \mathrm{~mL}$ of B27 [Invitrogen, 17504-044], $2 \mathrm{~mL}$ of N2 [Invitrogen, 17502-048], $2 \mathrm{~mL}$ of penicillin/streptomycin [Pen/Strep; Invitrogen], $2 \mathrm{~mL}$ L-glutamine [Invitrogen], $10 \mathrm{ng} / \mathrm{mL}$ mEGF [Peprotech, 315-09], 10 ng/mL hFGF2 [Peprotech, 100-18B]). MDA-MB-231, HepG2, and HEK293T cells were purchased from American Type Culture Collection. MDA-MB-231 cells were cultured in RPMI with 5\% FBS. HepG2 cells were cultured in MEM with $10 \%$ FBS. HEK293T cells were cultured in DMEM with 10\% FBS, sodium pyruvate, and nonessential amino acids. All cell types were cultured with $1 \times$ penicillin/streptomycin. Transient transfection was performed using Lipofectamine 2000 according to the manufacturer's protocols (Invitrogen).

Histology and immunofluorescent and immunohistochemistry staining of tissue sections

Tissues for histology were fixed in $4 \%$ paraformaldehyde, processed, and embedded in paraffin. Sections $(5 \mu \mathrm{m})$ were stained with hematoxylin and eosin, examined, and photographed using a Nikon TE 200 microscope. For immunostaining, paraffin sections were deparaffinized and rehydrated, and antigenic sites were unmasked using citric acid-based unmasking solution (Vector Laboratories, H-3300) in a Pascal pressure chamber (Dako), blocked in Superblock (Pierce, 37515) with 5\% normal goat serum for $1 \mathrm{~h}$ at room temperature, incubated with primary antibodies in block solution with $0.1 \%$ Triton X-100, washed four times for 5 min each in PBS, and incubated with immunofluorescently labeled secondary antibodies (Jackson ImmunoResearch) diluted in Superblock solution with 0.1\% Triton X-100. Stained slides were analyzed using a Nikon TE 200 microscope with a CoolSnap HQ digital camera. The sections were immunostained using Rb anti-Sox2 (Chemicon, AB5603), Rb anti-Yap1 (Cell Signaling 4912), and Rb anti-S100 $\beta$ (Novus NB110-57478). VectaStain Elite $\mathrm{ABC}$ kit (Vector Laboratories) was used for immunohistochemistry staining.

\section{In vitro kinase assay}

LATS1 phosphorylation assays were performed as described in Meng et al. (2015) with minor modifications. A truncated form of human LATS1 (amino acids 638-1130) was expressed as a GST fusion in Escherichia coli Rosetta cells and purified using glutathione Sepharose chromatography. Full-length Flag-MST2, Flag-MARK3, and V5-DLG5 proteins were expressed in
HEK293T cells and purified using anti-Flag M2 or anti-V5 affinity agarose gels (Sigma). Nonspecifically bound proteins were removed using high-salt washes $(1.15 \mathrm{M} \mathrm{NaCl})$, and Flag- or V5tagged proteins were released from beads using $3 \times$ Flag or $1 \times \mathrm{xV} 5$ peptides (Sigma). Proteins were incubated for $10 \mathrm{~min}$ at $30^{\circ} \mathrm{C}$ in kinase buffer containing $0.5 \mathrm{mM}$ ATP, $50 \mathrm{mM}$ Tris $-\mathrm{HCl} / \mathrm{pH}$ 7.5), $10 \mathrm{mM} \mathrm{MgCl} 2,2 \mathrm{mM} \mathrm{MnCl}, 0.1 \mathrm{mM}$ EDTA, 2 mM DTT, and $0.01 \%$ Brij 35 . MST-dependent phosphorylation of GSTLATS1 was detected by Western blotting with anti-phospho[Thr1079] LATS1 antibodies (Cell Signaling).

\section{Acknowledgments}

We thank Dr. Duojia Pan for the gift of Yap1 $1^{f l / f 1}$ mice, and Dr. Stefano Piccolo and Dr. Xiaolong Yang for the gift of plasmids. This work was supported by a bridge fund from the Fred Hutchinson Cancer Research Center; National Institutes of Health grants CA131047, CA188452, CA179914, and GM07270; and grants from the Ontario Research Fund (Global Leadership in Genomics and Life Sciences-GL2) and the Canadian Institutes of Health Research (MOP-133431). J.K., A.S., A.E., and V.V. conceived the study. L.A., H.M., A.E., and V.V. were responsible for the methodology. J.K., A.S., E.H.A., L.N., C.-C.C., S.R., and O.K. performed the investigation. J.K., A.E., and V.V. wrote the original draft of the manuscript. J.K., A.S., O.K., L.A., H.M., A.E., and V.V. reviewed and edited the manuscript. A.E. and V.V. acquired funding. L.A., H.M., A.E., and V.V. supervised the study.

\section{References}

Barry ER, Camargo FD. 2013. The Hippo superhighway: signaling crossroads converging on the Hippo/Yap pathway in stem cells and development. Curr Opin Cell Biol 25: 247-253.

Boggiano JC, Fehon RG. 2012. Growth control by committee: intercellular junctions, cell polarity, and the cytoskeleton regulate Hippo signaling. Dev Cell 22: 695-702.

Bryant DM, Mostov KE. 2008. From cells to organs: building polarized tissue. Nat Rev Mol Cell Biol 9: 887-901.

Chang Y, Klezovitch O, Walikonis RS, Vasioukhin V, LoTurco JJ. 2010. Discs large 5 is required for polarization of citron kinase in mitotic neural precursors. Cell Cycle 9: 1990-1997.

Chong YC, Mann RK, Zhao C, Kato M, Beachy PA. 2015. Bifurcating action of Smoothened in Hedgehog signaling is mediated by Dlg5. Genes Dev 29: 262-276.

Cordenonsi M, Zanconato F, Azzolin L, Forcato M, Rosato A, Frasson C, Inui $M$, Montagner $M$, Parenti AR, Poletti A, et al. 2011. The Hippo transducer TAZ confers cancer stem cell-related traits on breast cancer cells. Cell 147: 759-772.

Couzens AL, Knight JDR, Kean MJ, Teo G, Weiss A, Dunham WH, Lin Z-Y, Bagshaw RD, Sicheri F, Pawson T, et al. 2013. Protein interaction network of the mammalian Hippo pathway reveals mechanisms of kinase-phosphatase interactions. Sci Signal 6: rs15.

Dupont S, Morsut L, Aragona M, Enzo E, Giulitti S, Cordenonsi M, Zanconato F, Le Digabel J, Forcato M, Bicciato S, et al. 2011. Role of YAP/TAZ in mechanotransduction. Nature 474: 179-183.

Eng JK, McCormack AL, Yates JR. 1994. An approach to correlate tandem mass spectral data of peptides with amino acid sequences in a protein database. I Am Soc Mass Spectrom 5: 976-989. 
Enomoto M, Igaki T. 2011. Deciphering tumor-suppressor signaling in flies: genetic link between Scribble/Dlg/Lgl and the Hippo pathways. J Genet Genomics 38: 461-470.

Friedrichs F, Stoll M. 2006. Role of discs large homolog 5. World J Gastroenterol 12: 3651-3656.

Genevet A, Tapon N. 2011. The Hippo pathway and apico-basal cell polarity. Biochem J 436: 213-224.

Graus-Porta D, Blaess S, Senften M, Littlewood-Evans A, Damsky C, Huang Z, Orban P, Klein R, Schittny JC, Muller U. 2001. $\beta 1$-class integrins regulate the development of laminae and folia in the cerebral and cerebellar cortex. Neuron 31: 367-379.

Harvey KF, Pfleger CM, Hariharan IK. 2003. The Drosophila Mst ortholog, hippo, restricts growth and cell proliferation and promotes apoptosis. Cell 114: 457-467.

Hauri S, Wepf A, van Drogen A, Varjosalo M, Tapon N, Aebersold R, Gstaiger M. 2013. Interaction proteome of human Hippo signaling: modular control of the co-activator YAP1. Mol Syst Biol 9: 713.

Hossain Z, Ali SM, Ko HL, Xu J, Ng CP, Guo K, Qi Z, Ponniah S, Hong W, Hunziker W. 2007. Glomerulocystic kidney disease in mice with a targeted inactivation of Wwtr1. Proc Natl Acad Sci 104: 1631-1636.

Huang H-L, Wang S, Yin M-X, Dong L, Wang C, Wu W, Lu Y, Feng M, Dai C, Guo X, et al. 2013. Par-1 regulates tissue growth by influencing hippo phosphorylation status and hippo-salvador association. PLOS Biol 11: e1001620.

Kislinger T, Rahman K, Radulovic D, Cox B, Rossant J, Emili A. 2003. PRISM, a generic large scale proteomic investigation strategy for mammals. Mol Cell Proteomics 96: 106.

Klezovitch O, Vasioukhin V. 2015. Cadherin signaling: keeping cells in touch. F1000Research 4: 550.

Kwan JHM, Emili A. 2016. Simple and effective affinity purification procedures for mass spectrometry-based identification of protein-protein interactions in cell signaling pathways. Methods Mol Biol 1394: 181-187.

Lai D, Ho KC, Hao Y, Yang X. 2011. Taxol resistance in breast cancer cells is mediated by the hippo pathway component TAZ and its downstream transcriptional targets Cyr61 and CTGF. Cancer Res 71: 2728-2738.

Mak AB, Ni Z, Hewel JA, Chen GI, Zhong G, Karamboulas K, Blakely K, Smiley S, Marcon E, Roudeva D, et al. 2010. A lentiviral functional proteomics approach identifies chromatin remodeling complexes important for the induction of pluripotency. Mol Cell Proteomics 9: 811-823.

Martin-Belmonte F, Perez-Moreno M. 2012. Epithelial cell polarity, stem cells and cancer. Nat Rev Cancer 12: 23-38.

Meng Z, Moroishi T, Mottier-Pavie V, Plouffe SW, Hansen CG, Hong AW, Park HW, Mo J-S, Lu W, Lu S, et al. 2015. MAP4K family kinases act in parallel to MST1/2 to activate LATS1/2 in the Hippo pathway. Nat Commun 6: 8357.

Mo J-S, Park HW, Guan K-L. 2014. The Hippo signaling pathway in stem cell biology and cancer. EMBO Rep 15: 642656.

Mohseni M, Sun J, Lau A, Curtis S, Goldsmith J, Fox VL, Wei C, Frazier M, Samson O, Wong K-K, et al. 2014. A genetic screen identifies an LKB1-MARK signalling axis controlling the Hippo-YAP pathway. Nat Cell Biol 16: 108-117.

Moroishi T, Park HW, Qin B, Chen Q, Meng Z, Plouffe SW, Taniguchi K, Yu F-X, Karin M, Pan D, et al. 2015. A YAP/TAZ-induced feedback mechanism regulates Hippo pathway homeostasis. Genes Dev 29: 1271-1284.

Nechiporuk T, Fernandez TE, Vasioukhin V. 2007. Failure of epithelial tube maintenance causes hydrocephalus and renal cysts in Dlg5 $5^{-/-}$mice. Dev Cell 13: 338-350.
Nechiporuk T, Klezovitch O, Nguyen L, Vasioukhin V. 2013. Dlg5 maintains apical aPKC and regulates progenitor differentiation during lung morphogenesis. Dev Biol 377: 375-384.

Ni L, Li S, Yu J, Min J, Brautigam CA, Tomchick DR, Pan D, Luo X. 2013. Structural basis for autoactivation of human Mst2 kinase and its regulation by RASSF5. Structure 21: 1757-1768.

Oh S, Lee D, Kim T, Kim TS, Oh HJ, Hwang CY, Kong YY, Kwon KS, Lim DS. 2009. Crucial role for Mst1 and Mst2 kinases in early embryonic development of the mouse. Mol Cell Biol 29: 6309-6320.

Park R, Moon UY, Park JY, Hughes LJ, Johnson RL, Cho S-H, Kim S. 2016. Yap is required for ependymal integrity and is suppressed in LPA-induced hydrocephalus. Nat Commun 7: 10329.

Reilly E, Changela N, Naryshkina T, Deshpande G, Steward R. 2015. Discs large 5, an essential gene in Drosophila, regulates egg chamber organization. G3 (Bethesda) 5: 943952.

Shannon P, Markiel A, Ozier O, Baliga NS, Wang JT, Ramage D, Amin N, Schwikowski B, Ideker T. 2003. Cytoscape: a software environment for integrated models of biomolecular interaction networks. Genome Res 13: 2498-2504.

Sowa ME, Bennett EJ, Gygi SP, Harper JW. 2009. Defining the human deubiquitinating enzyme interaction landscape. Cell 138: 389-403.

Stoll M, Corneliussen B, Costello CM, Waetzig GH, Mellgard B, Koch WA, Rosenstiel P, Albrecht M, Croucher PJP, Seegert D, et al. 2004. Genetic variation in DLG5 is associated with inflammatory bowel disease. Nat Genet 36: 476-480.

Sun Y, Pollard S, Conti L, Toselli M, Biella G, Parkin G, Willatt L, Falk A, Cattaneo E, Smith A. 2008. Long-term tripotent differentiation capacity of human neural stem (NS) cells in adherent culture. Mol Cell Neurosci 38: 245-258.

Szymaniak AD, Mahoney JE, Cardoso WV, Varelas X. 2015. Crumbs3-mediated polarity directs airway epithelial cell fate through the Hippo pathway effector Yap. Dev Cell 34: 283-296.

Tallquist MD, Soriano P. 2000. Epiblast-restricted Cre expression in MORE mice: a tool to distinguish embryonic vs. extra-embryonic gene function. Genesis 26: 113-115.

Tian Y, Kolb R, Hong J-H, Carroll J, Li D, You J, Bronson R, Yaffe MB, Zhou J, Benjamin T. 2007. TAZ promotes PC2 degradation through a SCF $\beta-$ Trcp E3 ligase complex. Mol Cell Biol 27: 6383-6395.

Tomiyama L, Sezaki T, Matsuo M, Ueda K, Kioka N. 2015. Loss of Dlg5 expression promotes the migration and invasion of prostate cancer cells via Girdin phosphorylation. Oncogene 34: 1141-1149.

Wang SH, Celic I, Choi SY, Riccomagno M, Wang Q, Sun LO, Mitchell SP, Vasioukhin V, Huganir RL, Kolodkin AL. 2014. Dlg5 regulates dendritic spine formation and synaptogenesis by controlling subcellular N-cadherin localization. J Neurosci 34: $12745-12761$

Wu S, Huang J, Dong J, Pan D. 2003. hippo encodes a Ste-20 family protein kinase that restricts cell proliferation and promotes apoptosis in conjunction with salvador and warts. Cell 114: $445-456$.

Yang C-C, Graves HK, Moya IM, Tao C, Hamaratoglu F, Gladden AB, Halder G. 2015. Differential regulation of the Hippo pathway by adherens junctions and apical-basal cell polarity modules. Proc Natl Acad Sci 112: 1785-1790. 
DLG5 links Hippo and polarity protein networks

Yu F-X, Guan K-L. 2013. The Hippo pathway: regulators and regulations. Genes Dev 27: 355-371.

Zhang N, Bai H, David KK, Dong J, Zheng Y, Cai J, Giovannini M, Liu P, Anders RA, Pan D. 2010. The Merlin/NF2 tumor suppressor functions through the YAP oncoprotein to regulate tissue homeostasis in mammals. Dev Cell 19: 27-38.

Zhao B, Wei X, Li W, Udan RS, Yang Q, Kim J, Xie J, Ikenoue T, Yu J, Li L, et al. 2007. Inactivation of YAP oncoprotein by the Hip- po pathway is involved in cell contact inhibition and tissue growth control. Genes Dev 21: 2747-2761.

Zhao B, Ye X, Yu J, Li L, Li W, Li S, Yu J, Lin JD, Wang C-Y, Chinnaiyan AM, et al. 2008. TEAD mediates YAP-dependent gene induction and growth control. Genes Dev 22: 19621971.

Zhou Z, Guo Y, Liu Y, Zhang F, Wang Y, Shen B, Qin Y, Qiu J. 2015. Methylation-mediated silencing of Dlg5 facilitates bladder cancer metastasis. Exp Cell Res 331: 399-407. 


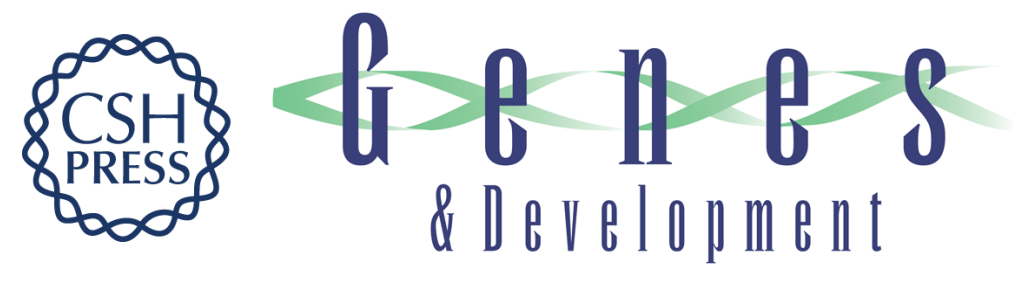

\section{DLG5 connects cell polarity and Hippo signaling protein networks by linking PAR-1 with MST1/2}

Julian Kwan, Anna Sczaniecka, Emad Heidary Arash, et al.

Genes Dev. 2016, 30:

Access the most recent version at doi:10.1101/gad.284539.116

\section{Supplemental Material \\ http://genesdev.cshlp.org/content/suppl/2017/01/13/30.24.2696.DC1}

References

This article cites 49 articles, 18 of which can be accessed free at: http://genesdev.cshlp.org/content/30/24/2696.full.html\#ref-list-1

Creative This article is distributed exclusively by Cold Spring Harbor Laboratory Press for the first Commons License

Email Alerting Service six months after the full-issue publication date (see http://genesdev.cshlp.org/site/misc/terms.xhtml). After six months, it is available under a Creative Commons License (Attribution-NonCommercial 4.0 International), as described at http://creativecommons.org/licenses/by-nc/4.0/.

Receive free email alerts when new articles cite this article - sign up in the box at the top right corner of the article or click here.

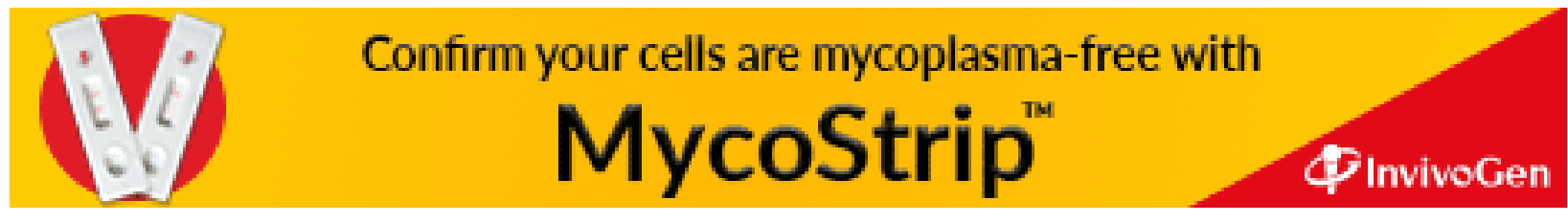

